\title{
Airway responsiveness and peak flow variability in the diagnosis of asthma for epidemiological studies
}

\author{
S.A. Lewis*, S.T. Weiss", J.R. Britton*
}

\begin{abstract}
Airway responsiveness and peak flow variability in the diagnosis of asthma for epidemiological studies. S.A. Lewis, S.T. Weiss, J.R. Britton. (C)ERS Journals Ltd 2001.

ABSTRACT: Airway responsiveness and variability in peak expiratory flow (PEF) are widely used as objective diagnostic measures of asthma, but it is not clear how these variables should be calculated or adjusted to obtain the highest diagnostic validity for physician-diagnosed asthma in the community.

Data from a community-based sample of 1,513 adults has been used. Airway responsiveness to methacholine and 7-day PEF data were obtained in 1991, asthma and respiratory symptoms were diagnosed by questionnaires in 1991 and 1999. Airway responsiveness was expressed as the provocative dose causing a $20 \%$ fall in forced expiratory volume in one second (PD20), two-point and least-squares regression slopes. PEF variability was expressed as daily amplitude, weekly standard deviation and mean of the two lowest readings. Continuous measures were adjusted for measures of baseline airway calibre by linear regression.

Measures of airway responsiveness had greater sensitivity for specificity for selfreported diagnosed asthma than expressions of PEF variability, before and after adjustment for airway calibre. Diagnostic validity was substantially better in adults aged $<50$ yrs; PD20 provided the best sensitivity for specificity $(61 \%$ for $95 \%$ at $\mathbf{8 . 3} \boldsymbol{\mu m o l})$. In those aged $\geqslant \mathbf{5 0} \mathrm{yrs}$, no measure was closely related to diagnosed asthma.

In younger age groups, provocative dose causing a $20 \%$ fall in forced expiratory volume in one second provides a valuable objective measure of asthma for epidemiological studies, but is unable to distinguish between asthma and chronic obstructive pulmonary disease in older people.
\end{abstract}

Eur Respir J 2001; 18: 921-927.

*Division of Respiratory Medicine, Clinical Sciences Building, City Hospital, University of Nottingham, UK. ${ }^{\#}$ Channing Laboratory, Dept of Medicine, Brigham and Women's Hospital, Boston, USA.

Correspondence: S.A. Lewis, Division of Respiratory Medicine, Clinical Sciences Building, City Hospital, Hucknall Road, Nottingham, NG5 1PB, UK. Fax: 441158404771

Keywords: Asthma epidemiology bronchial reactivity peak flow variability

Received: February 22001

Accepted after revision June 142001

This study was funded by the UK National Asthma Campaign and the British Lung Foundation. S. Lewis was funded by the Medical Research Council.

Objective and valid diagnostic methods are fundamental to the study of asthma. Measurements of airway hyperresponsiveness (AHR) and variability in peak expiratory flow (PEF) have been most widely used in this context. AHR generally appears to be more closely related to clinically-diagnosed asthma than PEF variability [1], but both measures lack specificity at acceptable levels of sensitivity in comparison with ascertaining disease using a questionnaire. Self-reported asthma has good sensitivity and specificity when tested against physician's opinion in developed societies [2]. However, it is likely to be less satisfactory for contrasting disease prevalence with developing societies, where access to health services may limit diagnostic ascertainment and linguistic or literacy divides may hinder comparability of symptom-based questionnaires. It is therefore important to explore methods of improving the diagnostic performance of available measures, which do not involve a questionnaire, including AHR and PEF variability. One variable that is closely related to both AHR and PEF variability is baseline lung function, and this study has been carried out to determine whether adjustment for baseline function improves the diagnostic validity of these measures.

The most current methods of measuring AHR and
PEF variability already include some degree of lung function adjustment, since AHR is usually expressed in terms of percentage change in the forced expiratory volume in one second (FEV1) and PEF variation as a function of the mean of PEF measures made during a period of recording. However, the authors have previously reported that AHR in the general population is still strongly and independently associated with baseline FEV1, expressed in absolute terms, as the per cent predicted and as the per cent of the forced vital capacity (FVC) [3]. Several studies have demonstrated that adjusting measures of AHR for baseline airway calibre may reduce age disparities in the distribution of AHR [3-5]. The question, therefore, arises whether the expression of AHR or PEF variability, as residual measures, after full correction for these different indices of initial lung function, improve the clinical diagnosis of asthma and help to distinguish between asthma and chronic obstructive airways disease, particularly in older people.

The authors used cross-sectional and longitudinal data from a general population sample, collected in a study of the relation between diet and lung disease, which was initially carried out in 1991 [6]. A follow-up questionnaire was carried out in 1999 to: 1) explore the relationship between doctor-diagnosed asthma 
and various different methods of expressing AHR and PEF variability; 2) to investigate the effects of adjustment for baseline lung function on these measures; and 3) to determine their relative predictive value for incident asthma over 9 yrs of follow-up. Whether these results were dependent on age was investigated by looking at these effects within three age strata.

\section{Methods}

Data were obtained from a previously described cohort of 2,633 subjects aged 18-70 yrs. The subjects were identified by systematic sampling from a random starting point in the electoral register of an area in the Nottingham Local Authority, and were first studied in 1991 [6]. All subjects undertook measurements of (prebronchodilator) FEV1 and FVC, and provided self-reported information on doctor-diagnosed asthma, age at onset of asthma and on the occurrence of respiratory symptoms over the previous 12 months (using questions from the International Union against Tuberculosis and Lung Disease (IUATLD) (1984) questionnaire [7]). Allergen skin sensitization to Dermatophagoides pteronyssinus, cat fur and grass pollen was measured, and the response defined as the mean of two right-angled weal diameters, one of which was the largest measurable diameter of the weal, excluding pseudopods and flares. The method of YAN et al. [8] was used to measure airway reactivity to methacholine in all 2,415 (92\%) consenting subjects with no medical contraindication to testing and who had a baseline FEV $1>60 \%$ pred or $1.5 \mathrm{~L}$, whichever was lower. Methacholine was given until FEV1 had fallen by $\geqslant 20 \%$ from the postsaline baseline, or up to a maximum cumulative dose of $12.25 \mu \mathrm{mol}$. All subjects were asked to keep a record of PEF measurements taken at 2 -h intervals when possible during waking hours for 7 consecutive days. The authors used data from the 1,664 diaries (from a total of 1,702 $(65 \%)$ returned $\mathrm{PEF}$ records) that included at least two readings on at least 4 of the last 5 days. By these criteria, a total of 1,513 subjects provided complete data on all of the variables mentioned earlier.

\section{Follow-up study}

In 1999, a self-completion questionnaire, comprising all of the original questions, was sent to subjects who were still alive and remained at their 1991 address or were traceable through their medical records to a more recent address. A short questionnaire was also sent to the General Practitioners (GPs) of subjects who reported doctor-diagnosed asthma in either 1991 or 1999, which asked whether a diagnosis of asthma was recorded in the patient's notes and whether it was first recorded before or after 1991.

\section{Analysis}

The following definitions were used for analysis. Doctor-diagnosed asthma: a history of asthma reported by the subject to have been confirmed by a doctor in 1991; incident asthma: doctor-diagnosed asthma reported only in 1999, with the first attack reported to have occurred after 1991; asthma-like symptoms: wheeze, tightness in the chest, nocturnal or exercise-induced dyspnoea, or nocturnal cough reported in 1991; and symptomatic asthma: diagnosed asthma with asthma-like symptoms. Airway responsiveness was calculated in the following three ways: 1) the inhaled dose of methacholine provoking a $20 \%$ fall in FEV1 (PD20) calculated by linear interpolation on a log dose-response plot; 2) the two-point slope (TPS) [9], calculated as the percentage fall between the postsaline FEV1 and the last dose of methacholine divided by the total dose of methacholine given; and 3) the least-squares regression slope (LSRS), calculated from all data points except the postsaline FEV1 by fitting a line of the form per cent fall in FEV $1=\alpha+\beta$ (dose) to compute $\beta$, the regression slope [10]. Subjects given less than two doses of methacholine were excluded from this analysis.

PEF variability was calculated from data from days 3-7 on the 7-day diary card (days 1 and 2 were excluded to reduce any learning effect [11]) and expressed for each subject as: 1) the amplitude as a percentage of the mean PEF computed as the 5-day mean of the daily highest minus the daily lowest value, divided by the daily mean (amp\%mean) [12]; 2) the standard deviation of all available PEF measurements as a percentage of the 5-day mean ( $\mathrm{SD}^{\circ} \%$ mean) [12]; and 3 ) the mean of the two lowest PEF readings as a percentage of the 5-day mean (two-lowest \%mean) [13].

Baseline lung function was expressed as: 1) the absolute value of FEV1 (FEV1); 2) FEV1 as a percentage of FVC (FEV\%FVC); and 3 ) the residual of FEV1 regressed upon sex, age and height (FEVR). Atopy was defined as the occurrence of any salinecorrected allergen skin weal with a diameter of $\geqslant 3 \mathrm{~mm}$.

Operator curves were plotted to compare sensitivity for specificity for doctor-diagnosed asthma for each measure of AHR and PEF defined earlier. Sensitivity was computed at constant $95 \%$ specificity to facilitate comparison between measures in the whole sample, and also in three predefined age strata $(18-35,36-50$, and 51-70) with approximately equal numbers in each. Measures of PEF variability were approximately log normally distributed, and were therefore base 10 $\log$ transformed for analysis. A reciprocal (1/(TPS+10) and $1 /($ LSRS +10$))$ transformation was applied to the dose-response measures of airway responsiveness, this resulted in variables being closer to normally distributed and having greater stability of variance than the equivalent $\log$ transformations [10]. Using data on subjects without doctor-diagnosed asthma, the independent effects of FEV $1, \mathrm{FEV} \% \mathrm{FVC}$ and FEVR on each of these measures was assessed using multiple linear regression, fitting higher order polynomial terms as appropriate. The differences between observed and predicted values from these models were used as measures adjusted for lung function, and sensitivity for specificity compared with that for the unadjusted airway responsiveness and PEF variability values. PD20 values were not adjusted for baseline 
Table 1. - Baseline characteristics in 1991 of the whole study population, subjects with complete methacholine challenge and peak flow variability data, and 1999 responders

\begin{tabular}{lccc}
\hline & Study group 1991 & $\begin{array}{c}\text { Subjects with complete } \\
\text { data in } 1991\end{array}$ & Responders in 1999\# $^{\#}$ \\
\hline Total & 2633 & $1513(57 \%$ of total) & $1794(70 \%$ of survivors $)$ \\
Females & $1321(50.2)$ & $773(51.1)$ & $911(50.8)$ \\
Age yrs & $740(28.1)$ & $317(21.0)$ & $403(22.5)$ \\
$\quad 18-35$ & $979(37.2)$ & $587(38.8)$ & $704(39.2)$ \\
36-50 & $914(34.7)$ & $609(40.3)$ & $687(38.3)$ \\
51-70 & $1306(49.6)$ & $785(51.9)$ & $939(52.3)$ \\
Smoking & $597(22.7)$ & $283(18.7)$ & $343(19.1)$ \\
Lifetime nonsmoker & $236(9.0)$ & $122(8.1)$ & $144(8.0)$ \\
Current smoker & $211(8.0)$ & $106(7.0)$ & $127(7.1)$ \\
$\quad$ Self-reported doctor-diagnosed asthma & & \\
$\quad$ Symptomatic asthma* &
\end{tabular}

Data are presented as N (column \%). *: self-reported doctor-diagnosed asthma with current asthma-like symptoms; ${ }^{\#}$ : at least 83 died since 1991.

lung function since the analysis of censored data would involve using nonstandard regression techniques. In subjects without diagnosed asthma in 1991, the predictive value of all of these measures for incident asthma between 1991 and 1999 was examined. The distributions of sex, age group, smoking history, FEV1, atopy, and the occurrence of asthmalike symptoms was compared in subjects with extreme values of airway responsiveness and PEF variability before and after adjustment for lung function. A p-value of 0.05 was used for all the statistical analyses.

\section{Results}

Unadjusted measures of airway hyperresponsiveness and peak expiratory flow variability

The baseline characteristics, age, sex, smoking history and the prevalence of diagnosed asthma, of the original sample of 2,633 subjects studied in 1991 are shown in table 1 . These characteristics were similar for the 1,513 subjects who provided complete methacholine challenge and PEF data in 1991, except for a relative under-representation of the 18-35 age group. By 1999, at least 83 of the original cohort had died; a current address was identified for 2,350 subjects of the original sample, of whom $1,794(70 \%$ of the original sample) returned the self-completion questionnaire. Again, there was a slightly lower response in 1999 from those aged 18-35 in 1991 (table 1), a larger proportion of whom had moved from the area. Of those with self-reported doctor diagnosis of asthma in 1991 or $1999,90 \%$ responding in 1999 gave written consent for inspection of their GP notes, and in 95\% of these cases, the GP provided information on asthma diagnosis from the medical record.

In $1991,122(8.1 \%)$ subjects from the study population of 1,513 reported doctor-diagnosed asthma, and of the 72 who responded in $1999,57(79 \%)$ of their GPs confirmed both the diagnosis and that it was recorded before 1991. Only 23 individuals reported incident asthma between 1991-1999 (2.2\% of those without asthma in 1991). Of these, a diagnosis was confirmed by the GP in 17 ( $81 \%$ of those who replied), and was first documented in the medical record after 1991 in 16.

During methacholine challenge testing in 1991, inhaled doses of methacholine up to a cumulative total of $12.25 \mu \mathrm{mol}$ generated a fall in FEV1 of $\geqslant 20 \%$ (hence a measurable PD20 value) in 13\% of those tested. The TPS method provided a measure of airway responsiveness in all subjects tested, and the LSRS method in all but three. In the sample of 1,513 subjects, the sensitivity and specificity of a PD20 of $\leqslant 12.25 \mu \mathrm{mol}$ for self-reported doctor-diagnosed asthma were $61 \%$ and $91 \%$, respectively, and these estimates were broadly similar in the youngest two age bands but lower in those aged $>50$. At $95 \%$ specificity (a level achieved by all methods), sensitivity was similar for all three methods of expressing AHR but was highest for PD20 (table 2). Over the full range of specificities attainable within the available range of PD20 measurements, the operator curves for TPS and LSRS were also very similar to that for PD20 (fig. 1). Incident asthma between 1991-1999, which was rare overall, was significantly more common in those with

Table 2.-Sensitivity at $95 \%$ specificity of standard measures of airway reactivity and peak flow variability for doctor-diagnosed asthma

\begin{tabular}{lcccc}
\hline & \multicolumn{4}{c}{ Sensitivity at $95 \%$ specificity \% } \\
\cline { 2 - 5 } & All & $18-35$ & $36-50$ & $>50$ \\
\hline Airway reactivity & & & & \\
PD20 & 57 & 56 & 65 & 39 \\
Regression slope & 52 & 53 & 63 & 36 \\
Two-point slope & 54 & 53 & 65 & 39 \\
Peak flow variability & & & & \\
Amplitude\%mean & 25 & 6 & 41 & 21 \\
SD\%mean & 22 & 9 & 35 & 10 \\
Two-lowest\%mean & 20 & 6 & 39 & 8 \\
\hline
\end{tabular}

PD20: prouocative dose of methacholine causing a $20 \%$ fall in forced expiratory volume in one seond. 

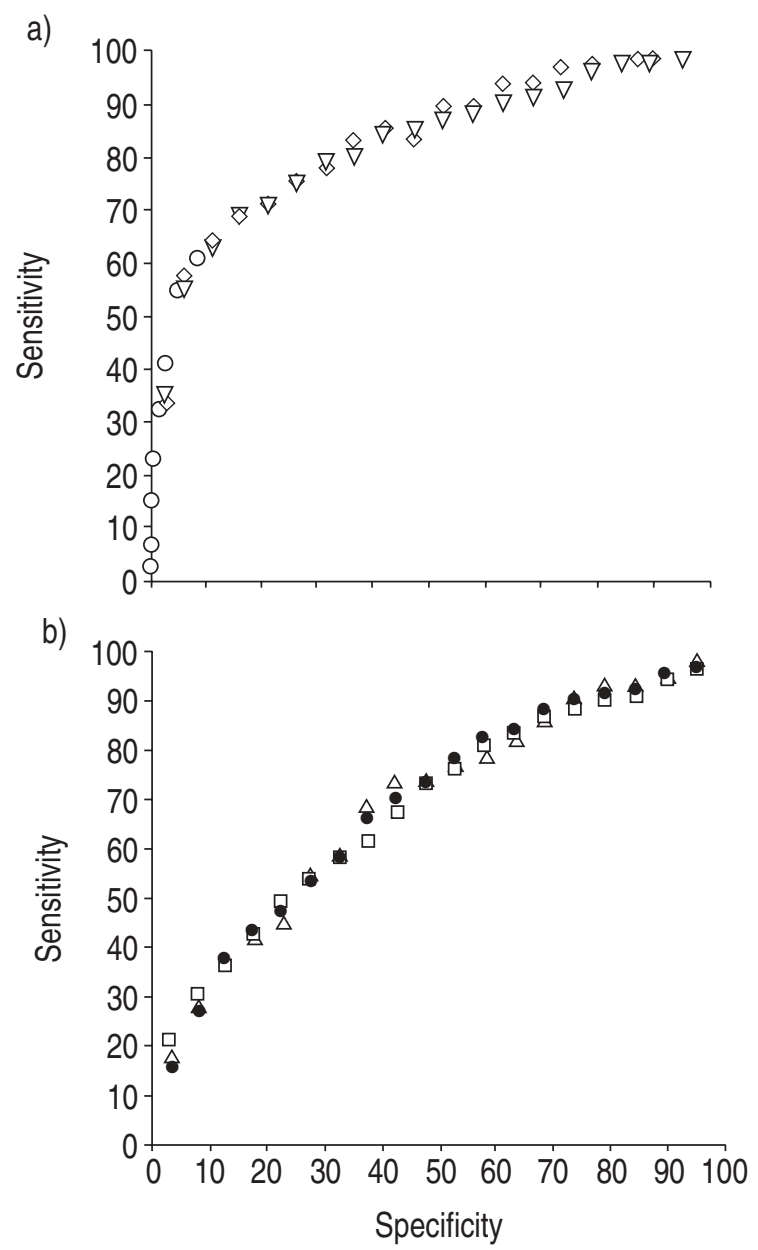

Fig. 1.-Operator curves of sensitivity versus specificity for measures of a) airway responsiveness and b) peak flow variability. $\diamond$ : two-point slope; $\nabla$ : regression slope; $\bigcirc$ : provocative dose of methacholine causing a $20 \%$ fall in forced expiratory volume in one second; $\square$ : amplitude $\%$ mean peak expiratory flow (PEF); $\triangle$ : SD\%mean PEF; 0 : two-lowest\%mean PEF.

a PD20 $<12.25 \mu \mathrm{mol}(5 \%$ and $2 \%$, respectively), but the predictive value of PD20 $<12.25 \mu$ mol was low at $22 \%$ sensitivity, $92 \%$ specificity.

For PEF variability, the amp $\%$ mean measure provided higher sensitivity at $95 \%$ specificity in the total sample and in most age groups than either the $\mathrm{SD} \%$ mean or the two-lowest $\%$ mean. However, even sensitivity for amp $\%$ mean was low at only $25 \%$ in the whole group and, at best, $41 \%$ in the age group 36-50 yrs (table 2). Amp \%mean was consistently and substantially less sensitive at all levels of specificity than any measure of AHR (fig. 1). At 95\% specificity, all of the PEF measures provided poor sensitivity for incident asthma between 1991-1999; greatest for amp $\%$ mean at $17 \%$ sensitivity.

\section{Adjustment for baseline lung function}

As previously shown for PD20 [3], absolute FEV1, $\mathrm{FEV} \% \mathrm{FVC}$, and FEVR were all significantly and independently associated with reciprocally transformed TPS and LSRS in both the whole group and after exclusion of subjects with doctor-diagnosed asthma (table 3). The effect of adjusting either AHR measurement for baseline lung function by these models was to reduce overall sensitivity at any level of specificity, especially in subjects aged $>35 \mathrm{yrs}$ (table 4).

Each measure of PEF variability was strongly and independently associated with FEV1, FEV\%FVC and FEVR (table 3). Adjustment for baseline airway calibre substantially weakened the relationship of PEF variability with diagnosed asthma, especially in subjects aged 36-50 yrs (table 4).

Table 4.-Sensitivity at $95 \%$ specificity for doctordiagnosed asthma of airway reactivity and peak flow variability adjusted for airway calibre

\begin{tabular}{lcccc}
\hline & \multicolumn{4}{c}{ Sensitivity at $95 \%$ specificity \% } \\
\cline { 2 - 5 } & All & $18-35$ & $36-50$ & $>50$ \\
\hline Airway reactivity & & & & \\
Regression slope & 47 & 53 & 54 & 36 \\
Two-point slope & 50 & 53 & 63 & 31 \\
Peak flow variability & & & & \\
Amplitude\%mean & 12 & 6 & 20 & 10 \\
SD\%mean & 11 & 3 & 14 & 10 \\
Two-lowest\%mean & 16 & 6 & 27 & 8 \\
\hline
\end{tabular}

Table 3. - Regression coefficients (SE) for the relationships listed with airway responsiveness and peak expiratory flow (PEF) variability in nonasthmatics (only statistically significant terms retained)

\begin{tabular}{|c|c|c|c|c|c|}
\hline & \multicolumn{2}{|c|}{ Airway responsiveness } & \multicolumn{3}{|c|}{ PEF variability } \\
\hline & LSRS & TPS & Amp $\%$ mean & $\mathrm{SD} \%$ mean & Two-lowest $\%$ mean \\
\hline Constant & $-0.02460(0.020)^{*}$ & $-0.04305(0.022)^{*}$ & $1.311^{\#}$ & $0.903^{\#}$ & $1.934^{\#}$ \\
\hline FEV1 & $0.00733(0.002) *$ & $0.00672(0.002)^{*}$ & $-0.06273(0.008)^{\#}$ & $-0.05445(0.008)^{\#}$ & $0.004041(0.001)^{\#}$ \\
\hline $\mathrm{FEV}_{1}^{2}$ & $-0.00088(0.000)^{*}$ & $-0.00077(0.000)^{*}$ & & & \\
\hline FEV \%FVC & $0.00235(0.001)^{\#}$ & $0.00279(0.001)^{\#}$ & $-0.00248(0.001)^{*}$ & $-0.00231(0.001)^{*}$ & $0.00021(0.000)^{*}$ \\
\hline $\mathrm{FEV}^{2} \% \mathrm{FVC}^{2}$ & $-0.00001(0.000)^{\#}$ & $-0.00002(0.000)^{\#}$ & & & \\
\hline FEVR & $0.00244(0.000)^{\#}$ & $0.00222(0.000)^{\#}$ & $-0.0365(0.008)^{\#}$ & $-0.05244(0.008)^{\#}$ & $0.00488(0.001)^{\#}$ \\
\hline FEVR $^{2}$ & $-0.00069(0.000)^{*}$ & $-0.00068(0.000) *$ & & & \\
\hline
\end{tabular}

RS: least-squares regression slope; TPS: two-point slope; FEV1: forced expiratory volume in one second; FVC: forced vital capacity; FEVR: the residual FEV1. *: $\mathrm{p}<0.05$; $^{\#}: \mathrm{p}<0.001$. 
Effect of adjustment for baseline lung function on the characteristics and predictive value of airway hyperresponsiveness

In view of the poor relationship of asthma with measurements of AHR in those aged $>50 \mathrm{yrs}$, and with PEF variability at all ages, this analysis was limited to AHR in subjects $<50$ yrs. In this age group, adjusting either TPS or LSRS for baseline airway calibre had little impact on the sex or age distribution of those with AHR. Adjustment increased the proportion that were atopic (from $62 \%$ to $66 \%$ ), and reduced the proportion that were current smokers (from $29 \%$ to $26 \%$ ), but only marginally. Adjustment made negligible difference to the association of AHR with incident asthma between 1991-1999, or to the occurrence of asthma-like symptoms.

Dose-response slope versus provocative dose of methacholine causing a 20\% fall in forced expiratory volume in one second

In the subjects aged $<50$ yrs, continuous doseresponse measurements provided little improvement in sensitivity for diagnosed asthma for reductions in specificity beyond that available for the PD20. To explore why this was the case, in a post hoc analysis, the dose-response slope measurement was categorized into 20 five-percentile categories, and the proportion with asthma in 1991, incident asthma up to 1999, and asthma-like symptoms according to increasing AHR was examined (fig. 2). There was a marked step-down in the occurrence of asthma and undiagnosed asthmalike symptoms at values below the 90th percentile (virtually equivalent to $95 \%$ specificity) of the doseresponse slope. Using the PD20 at this level of specificity (cut-off $\mathrm{PD} 20=8.3 \mu \mathrm{mol}$ ), the vast majority of subjects aged $<50$ with AHR (79 of $91(87 \%)$ ) reported either diagnosed asthma or asthma-like symptoms.

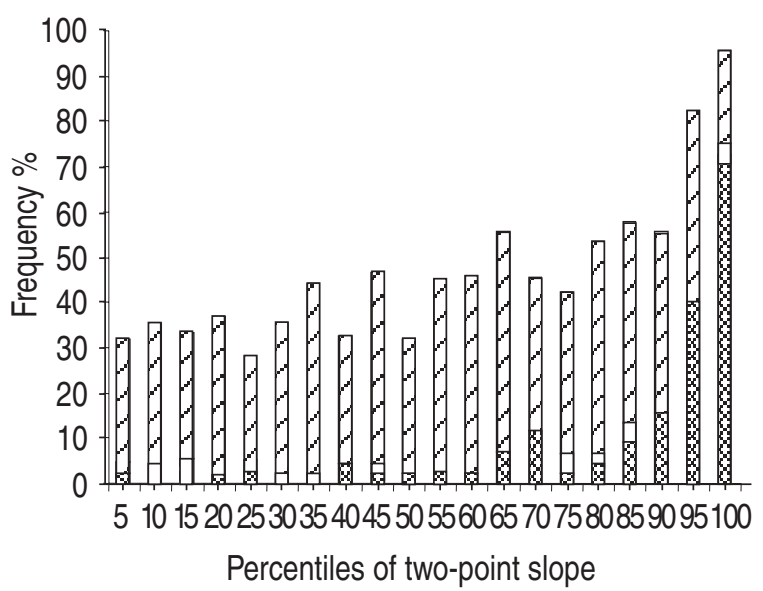

Fig. 2.-Diagnosed asthma (网), incident asthma ( $\square$ ) and asthmalike symptoms $(\mathbb{Z})$ according to percentiles of airway hyperresponsiveness two-point slope.

\section{Discussion}

The present study confirms that measurements of AHR are more closely approximate to diagnosed asthma in the community than measures of PEF variability in all age groups. However, sensitivity for specificity of airway responsiveness was substantially lower in older age groups, and was reduced further when adjustment was made for baseline airway calibre. In subjects aged $<50$, adjusting for baseline airway calibre made no substantial difference either to the diagnostic validity of measurements of AHR or the characteristics of the hyperresponsive group. In this younger age group, hyperresponsiveness defined by a cut-off achievable using the PD20 measurement, identified the majority of diagnosed asthmatics and was present in only a small minority of individuals who had neither diagnosed asthma nor symptoms characteristic of asthma.

In the present study, measurement of self-reported doctor-diagnosed asthma via a questionnaire was used as the "gold standard"; self-reported asthma has been previously shown to have good specificity, although it may have lower sensitivity, compared to a physician's opinion [2]. Specificity against GP records was found to be $\sim 80 \%$; discrepancies are likely to reflect incomplete GP records from early life. The GP records for subjects who did not report asthma on either occasion were not accessed, but the sensitivity of the authors' measurement of self-reported asthma is likely to have been increased by approaching each individual twice. Only 12 individuals reported asthma for the first time in 1999, but reported that it had started before 1991. If misclassification of asthma status tends to reflect the current clinical status of asthma, it is possible that the estimates of sensitivity relative to specificity of AHR and PEF variability may have been overestimated, but this is unlikely to have affected the comparison of the different measures. The response rate in 1999 was estimated at $70 \%$ but is likely to be higher than this, as an exhaustive search to identify deaths was not made. The response rate did not appear to reflect disease status and is therefore relatively unlikely to have affected the results. The majority $(89 \%)$ of asthmatics were symptomatic at the time of the study, and although no single symptom was specific for self-reported asthma within this population, wheezing in the past 12 months had the highest specificity for sensitivity (69\% sensitivity, 83\% specificity). Thus, the present study confirms that questionnaires provide a reliable and sensitive measurement of asthma within a developed community. However, in epidemiological comparisons of asthma prevalence between communities where there are linguistic or cultural divides, problems of literacy or access to health provision, objective methods that do not rely on self-report are still essential.

PEF variability is a measurement of airflow variability and should therefore be a close measurement of the inherent abnormality of asthma, but in practice it is difficult to estimate. Data was collected over 7 days and analysed over the last 5 days of recording to allow for learning effects [11]. It is possible that a longer period of recording would have 
resulted in more useful measurements, but this approach has to be weighed against the decreased availability of data arising from a longer period of recording. Only two-thirds of the subjects returned their weekly diaries with adequate readings for analysis, substantially less than the $92 \%$ of individuals who provided useable methacholine challenge data. A day of PEF readings was considered to be viable if there were at least two readings; the median number was, in fact, seven per day for all age ranges, except the youngest age group where the median number of readings was six. This may have contributed to the poorer diagnostic validity of PEF variability in younger individuals. Three different measurements of PEF variability were tried, which respectively measured variability within, and between, days and on isolated days of low PEF. All three measurements were generally more closely correlated to measures of baseline lung function than were measures of airway responsiveness, and this relationship appeared to largely explain, rather than weaken, the association of PEF variability with diagnosed asthma, especially in the older age groups. This is consistent with previous reports that PEF variability yields information on a different physiological component of disease to that measured by bronchial responsiveness [1, 14], but the data suggests that it may largely be a measure of airway calibre, and as a definitive measure of asthma status, is relatively uninformative. The data also suggests that techniques of PEF variability are essentially unworkable in community surveys.

The relationship between airway responsiveness and baseline lung function has been widely reported [3, 15-18] but is not fully understood. Explanations for this association range from the suggestion that small airways cause AHR because subjects with small lungs receive a proportionately greater dose of agonist during bronchial challenge, to the hypothesis that AHR is a fundamental abnormality, which causes impairment of FEV1 [19, 20]. Whatever the explanation, adjusting for airway calibre could either improve or impair the relationship between airway responsiveness and asthma. In some studies, making an adjustment for lung function to measures of airway responsiveness appears to alter the age and sex characteristics of those with hyperresponsiveness [3-5]. However, in the first of these studies, PEAT et al. [4] found little change in the sensitivity for specificity of the adjusted measure for asthma. The population used in the present study comprised a wider age range, and conversely, it was found that the diagnostic value of AHR was substantially reduced by adjusting for lung function in the older age group. The authors chose to subdivide arbitrarily at age $50 \mathrm{yrs}$, but the same was true in further analyses of the 50-60 yrs age group (data not presented), suggesting that 50 yrs provides a reasonable cut-off age beyond which measures of AHR, like PEF variability, are largely determined by lung function, and are, therefore, effectively markers of chronic obstructive pulmonary disease (COPD). In nonasthmatics aged $<50$, the relationship between AHR and lung function was relatively weak, even though it was statistically significant. Therefore, adjustment for lung function had little impact on either the sensitivity or the characteristics of those with AHR. In this younger age group, where the distinction between asthma and COPD does not present such a problem, measures of AHR are much closer to diagnosed asthma or to symptoms suggestive of asthma. Data was not available on subjects $<18$ yrs of age, hence it is not known whether this is also the case in children.

Historically, airway responsiveness has usually been expressed using the PD20, although dose-response alternatives were introduced more recently, with the intention of providing continuous measurements of airway responsiveness, consistent with the notion of disease as a continuum. The dose-response measurements have very similar repeatability to PD20 [10], but are more difficult to analyse. Even when reciprocal transformation is used, as in the present study, normally distributed variables are not produced, rendering statistical analysis complicated. It is evident from the analyses of these measures in those aged $<50$ yrs that airway responsiveness may bear a statistically significant relationship with asthma at levels below that achievable using PD20 [16], but there is relatively little gain in sensitivity for loss in specificity beyond this level. Moreover, over the range of specificities attainable using PD20, this measure had marginally better sensitivity for asthma than either dose-response measurement. A PD20 cutoff of $8.3 \mu \mathrm{mol}$ identified almost two-thirds of asthmatics $(63 \%$ of those confirmed by the GP), whilst the vast majority $(87 \%)$ of hyperresponsive individuals, had either asthma or asthma-like symptoms. PD20 is also relatively easy to compute.

Despite previous concerns about validity [21], the present study suggests that the provocative dose of methacholine causing a $20 \%$ fall in forced expiratory volume in one second provides the best nonquestionnaire diagnostic measurement of asthma.

Acknowledgements. The authors would like to thank I. Pavord, I. Wahedna, C. Wong, and W. Kinnear for assistance with the survey fieldwork, the General Practitioners and their colleagues who allowed the use of their premises for the study and the people of Gedling who took part.

\section{References}

1. Higgins BG, Britton JR, Chinn S, Cooper S, Burney PGJ, Tattersfield AE. Comparison of bronchial reactivity and peak expiratory flow variability measurements for epidemiologic studies. Am Rev Respir Dis 1992; 145: 588-593.

2. Toren K. Asthma and asthma-like symptoms in adults assessed by questionnaires. Chest 1993; 104: 600-608.

3. Britton J, Pavord I, Richards $\mathrm{K}$, et al. Factors influencing the occurrence of airway hyperreactivity in the general population: The importance of atopy and airway calibre. Eur Respir J 1994; 7: 881-887.

4. Peat JK, Salome CM, Xuan W. On adjusting measurements of airway responsiveness for lung size and airway caliber. Am J Respir Crit Care Med 1996; 154: $870-875$. 
5. Ulrik CS. Factors associated with increased bronchial responsiveness in adolescents and young adults: The importance of adjustment for prechallenge FEV1. J Allergy Clin Immunol 1996; 97: 761-767.

6. Britton J, Pavord I, Richards K, et al. Dietary magnesium, lung function, wheezing, and airway hyperreactivity in a random adult population sample. Lancet 1994; 344: 357-362.

7. Burney PGJ, Laitinen LA, Perdrizet S, et al. Validity and repeatability of the IUATLD (1984) bronchial symptoms questionnaire: an international comparison. Eur Respir J 1989; 2: 940-945.

8. Yan K, Salome C, Woolcock AJ. A rapid method for measurement of bronchial responsiveness. Thorax 1983; 38: 760-765.

9. O'Connor G, Sparrow D, Taylor D, Segal M, Weiss S. Analysis of dose-response curves to methacholine. An approach suitable for population studies. Am Rev Respir Dis 1989; 136: 1412-1417.

10. Chinn S, Burney PGJ, Britton JR, Tattersfield AE, Higgins BG. Comparison of PD20 with two alternative measures of response to histamine challenge in epidemiological studies. Eur Respir J 1993; 6: 670-679.

11. Britton J. Measurement of peak flow variability in community populations: methodology. Eur Respir $J$ 1997; 10: Suppl. 24, 42S-44S.

12. Higgins BG, Britton JR, Chinn S, Lai KK, Burney PGJ, Tattersfield AE. Factors affecting peak expiratory flow variability and bronchial reactivity in a random population sample. Thorax 1993; 48: 899-905.

13. Siersted HC, Mostgaard G, Hyldebrandt N, Hansen HS, Boldsen J, Oxhoj H. Interrelationships between diagnosed asthma, asthma-like symptoms, and abnormal airway behaviour in adolescence: The Odense Schoolchild Study. Thorax 1996; 51: 503-509.
14. Brand PLP, Postma DS, Kerstjens HAM, Koeter GH and The Dutch CNSLD Study Group. Relationship of airway hyperresponsiveness to respiratory symptoms and diurnal peak flow variation in patients with obstructive lung diseases. Am Rev Respir Dis 1991; 143: 916-921.

15. Dirksen A, Madsen F, Engel T, Frolund L, Heinig JH, Mosbech H. Airway calibre as a confounder in interpreting bronchial responsiveness in asthma. Thorax 1992; 47: 702-706.

16. Peat JK, Salome CM, Berry G, Woolcock AL. Relation of dose-response slope to respiratory symptoms and lung function in a population study of adults living in Busselton, West Australia. Am Rev Respir Dis 1992; 146: 860-865.

17. Ulrik CS. Bronchial responsiveness to inhaled histamine in both adults with intrinsic and extrinsic asthma: The importance of prechallenge forced expiratory volume in 1 second. J Allergy Clin Immunol 1993; 91: 120-126.

18. Rijcken B, Schouten JP, Weiss ST, Speizer FE, Van Der Lende R. The relationship between airway responsiveness to histamine and pulmonary function level in a random population sample. Am Rev Respir Dis 1988; 137: 826-832.

19. Parker DR, O'Connor GT , Sparrow D, Segal MR, Weiss ST. The relationship of nonspecific airway responsiveness and atopy to the rate of decline of lung function. Am Rev Respir Dis 1990; 141: 589-594.

20. Rijcken B, Schouten JP, Xu X, Rosner B, Weiss ST. Airway hyperresponsiveness to histamine associated with accelerated decline in FEV1. Am J Respir Crit Care Med 1995; 151: 1377-1382.

21. Britton J, Lewis S. Objective measures and the diagnosis of asthma. BMJ 1998; 317: 227-228. 